\title{
BMJ Open Effectiveness of influenza vaccination for preventing influenza-related complications in people with asthma: a systematic review protocol
}

\author{
Eleftheria Vasileiou, ${ }^{1}$ Aziz Sheikh, ${ }^{1}$ Chris Butler, ${ }^{2,3}$ Beatrix von Wissmann, ${ }^{4}$ \\ Jim McMenamin, ${ }^{4}$ Lewis Ritchie, ${ }^{5}$ Lilly Tian, ${ }^{6}$ Colin Simpson ${ }^{1}$
}

To cite: Vasileiou $\mathrm{E}$ Sheikh A, Butler C, et al. Effectiveness of influenza vaccination for preventing influenza-related complications in people with asthma: a systematic review protocol. BMJ Open 2016;6: e010133. doi:10.1136/ bmjopen-2015-010133

- Prepublication history and additional material is available. To view please visit the journal (http://dx.doi.org/ 10.1136/bmjopen-2015010133).

Received 20 October 2015 Revised 3 February 2016 Accepted 10 February 2016

CrossMark

For numbered affiliations see end of article.

Correspondence to Eleftheria Vasileiou; E.Vasileiou@ed.ac.uk

\begin{abstract}
Introduction: Influenza vaccination is administered annually as a preventive measure against influenza infection and influenza-related complications in highrisk individuals, such as those with asthma. However, the effectiveness of influenza vaccination in people with asthma against influenza-related complications is still not well established.
\end{abstract}

Methods and analysis: We will search the following databases: MEDLINE (Ovid), EMBASE (Ovid), Cumulative Index to Nursing and Allied Health Literature (CINAHL), Cochrane Central Register of Controlled Trials (CENTRAL), Scopus, Cochrane Database of Systematic Reviews (CDSR), Web of Science Core Collection, Science direct, WHO Library Information System (WHOLIS), Global Health Library and Chinese databases (CNKI, Wanfang and ChongQing VIP) from Jan 1970 to Jan 2016 for observational and experimental studies on effectiveness of influenza vaccine in people with asthma. The identification of studies will be complemented with the searching of the reference lists and citations, and contacting influenza vaccine manufacturers to identify unpublished or ongoing studies. Two reviewers will extract data and appraise the quality of each study independently. Separate meta-analyses will be undertaken for observational and experimental evidence using fixed-effect or random-effects models, as appropriate.

Ethics and dissemination: Formal ethical approval is not required, as primary data will not be collected. The review will be disseminated in peer-reviewed publications and conference presentations.

\section{INTRODUCTION}

Influenza is an acute respiratory illness caused by infection with the influenza virus. Influenza can cause respiratory tract infections and results in complications, which can result in substantial morbidity and in some cases death. ${ }^{12}$

Worldwide, influenza causes an estimated five million cases of severe illness and half a

\section{Strengths and limitations of this study}

- Systematic review of high-quality studies, such as randomised controlled trials.

- The selection of studies, data extraction, and the quality assessment will be conducted by two independent authors.

- The protocol has been created according to published PRISMA-P guidelines.

- We do not have any language limitations.

- The inclusion of non-randomised studies may produce evidence of possible bias. Sensitivity analysis is planned to be conducted to reduce the risk of poor evidence, by excluding studies at high risk of bias.

million deaths each year. In the USA, for example, the overall cost to health services is estimated at US $\$ 87$ billion per annum. ${ }^{3} 4$ The burden of influenza is particularly high in individuals with chronic medical conditions. Pneumonia and influenza-related mortality rates range from less than 10/100 000 in healthy adults to over $600 / 100000$ in adults with chronic illness. ${ }^{5}$ In the USA, during the influenza seasons 2004-2012, among 794 flu-associated deaths in children with or without high-risk medical conditions, $127(16 \%)$ had asthma. ${ }^{6}$ It is estimated that $8 \%$ of asthma exacerbations are caused by influenza. ${ }^{7}$ Groups at increased risk of severe influenza include those with asthma, other chronic diseases, pregnant women, morbid obesity, individuals over the age of 65 years, children aged 6 months to 4 years, residents of nursing homes or chronic care facilities, and their carers. 589

The Global Initiative for Asthma definition for asthma is described as the history of respiratory symptoms, such as wheeze, shortness of breath, chest tightness and cough that vary over time and in intensity, together 
with variable expiratory airflow limitation. ${ }^{10}$ The influenza virus is a trigger that causes inflammation in the airways of people with asthma resulting in severe complications, such as exacerbation of asthma, hospitalisation, or even death. Thus, people with asthma are at risk of developing influenza-related complications due to their sensitive airway system. ${ }^{6}{ }^{11}$

Annual immunisation with influenza vaccine of people with asthma, is the current preventive measure against seasonal influenza and an influenza-triggered asthma attack, ${ }^{12}$ which is recommended by WHO and many national immunisation technical advisory groups. ${ }^{9}{ }^{13-16}$ According to the UK guidelines, people with asthma who require continuous or repeated use of inhaled or systemic steroids, or with previous exacerbations requiring hospital admission, are considered as having severe asthma, and free influenza vaccination is offered. ${ }^{9}$ However, this varies by country. In addition, immunoresponse to the influenza vaccine may be adversely affected in people with asthma on high-dose inhaled corticosteroid therapy. However, further evidence is needed regarding the impact of corticosteroids on immunity induced by the influenza vaccine. ${ }^{17}$

Influenza vaccines currently available worldwide include: (1) whole virion-inactivated vaccines; (2) subunit-inactivated vaccines which contain only surface antigens ( $\mathrm{H}$ and $\mathrm{N})$; (3) split virion-inactivated vaccines which contain surface and internal antigens; and (4) live-attenuated, cold-adapted vaccines in which the live virus can only multiply in the cooler nasal passages. ${ }^{18}$

Vaccination is believed to induce a variable degree of protection (ranging from 0 to $80 \%$ ) against influenza and its complications. The high mutation of the influenza virus and the emergence of new strains, the lack of carryover protection by previous infection or vaccination, are some reasons why annual repetition is required. ${ }^{10} 19{ }^{20}$ Additionally, a good antigenic match between the circulating influenza virus and the strains included in the vaccine is required for optimal protection. ${ }^{21}$ Thus, regular monitoring of the effectiveness of influenza vaccination is needed.

Experimental studies and, in particular, randomised controlled trials (RCT) are considered the best evidence for evaluating influenza vaccines. However, there are feasibility issues raised when conducting clinical trials for vaccine efficacy evaluation. These include the costs involved in the conduct of RCTs. For example, the average coverage cost per patient enrolment in clinical trials in the UK was $£ 9900$ in $2007 .{ }^{22}$ Additionally, each influenza season should be monitored as the virulence circulating influenza virus types are highly variable and unpredictable. Ethical issues may also be raised when vaccine effectiveness is assessed in placebo-controlled trials, as the influenza vaccine is a recommended product particularly in clinically high-risk individuals, such as those with asthma, for the prevention of influenza-related complications. ${ }^{23-26}$ Most RCTs have, therefore, been conducted in healthy populations, and only a few in people with asthma or other medical conditions. ${ }^{27-30}$ Therefore, it is necessary to include observational studies (despite their poorer quality, compared to RCTs) as a source of evidence for the evaluation of influenza vaccines among high-risk groups. $^{23-25}$ Additionally, the test-negative (TND) (observational case-control study) design is considered to be a good measure of vaccine effectiveness. This is due to the use of the highly specific laboratory real-time polymerase chain reaction (RT-PCR) testing to confirm (or refute) influenza among a population exposed or not exposed to influenza vaccine. ${ }^{31} 32$ Although these TND studies only assess whether the vaccine is an effective intervention against laboratory-confirmed influenza, this assessment of vaccine effectiveness is an important public health priority, particularly for at-risk people of influenza-related complications. ${ }^{633}$

A systematic review carried out by Cates $e t$ al in 2013, evaluated the effectiveness of influenza vaccination in people with asthma. The primary outcome for the Cates et al review was laboratory-confirmed influenza-related asthma exacerbations. This review found one RCT in children and none in adults. They were therefore unable to conclude that influenza vaccination protected people with asthma from asthma exacerbations related to influenza infection. ${ }^{34}$ Our planned approach builds on the review by Cates $e t a l$, by also including TND studies (as well as RCTs) with laboratory-confirmed influenza outcomes, and other observational study designs with non-specific clinical outcomes (influenza-like illness, or any other respiratory or cardiovascular and cerebrovascular illness).

\section{OBJECTIVES}

We seek to evaluate the effectiveness of influenza vaccination in preventing influenza infection, and influenza-related complications in people with asthma.

\section{METHODS}

Design

We will conduct a systematic review of published and unpublished research reports.

\section{Eligibility criteria}

Types of studies

The following experimental and observational study designs will be eligible for inclusion:

- RCTs or controlled clinical trials

- TND studies and cohort (prospective and retrospective), case-control studies and nested case-control studies.

Systematic reviews and meta-analyses will be used only for identifying additional studies; these will not be included in this review.

\section{Types of participants}

According to international and national health organisations, children and adults with chronic underlying 
conditions are at high risk of developing severe influenza-related complications. ${ }^{9} 11$ Thus, annual influenza vaccination is recommended in people with asthma.

We will include papers which have adopted definitions reflecting the heterogeneous definition of asthma. ${ }^{10}$ These will include people with a history of asthma symptoms and evidence of reversible obstruction of respiratory airways (eg, bronchodilator reversibility tests or other tests) in the preceding 12 months, confirmed by a physician. Patients with asthma will be included in the review regardless of their current prescribed treatment for asthma.

People with a diagnosis of asthma will be included in the review. Additionally, if participants in a study have asthma or some other chronic medical conditions (eg, chronic respiratory disease, chronic heart disease, chronic liver disease, chronic kidney disease, chronic neurological disease, diabetes, immunosuppression, asplenia, or dysfunction of spleen), they will still be included in the review, provided that separate data on asthma are available from the study, or through personal communication with the authors.

\section{Types of intervention}

Studies including any of the licensed seasonal influenza vaccines. Studies that assess the effectiveness of pandemic influenza vaccine will be excluded. We will include influenza vaccines of any type, any dose, and any schedule, when compared with placebo, no vaccine or other vaccine:

- Inactivated or live attenuated influenza vaccines

- Recombinant influenza vaccines

- Virosomal vaccines

- Whole, subunit, or split vaccines

- Monovalent, bivalent, trivalent, quadrivalent or polyvalent.

\section{Types of outcome measures}

Influenza and asthma-related outcomes will be included in this review. In addition, the long-term benefits of the influenza virus vaccine (during the influenza season) and the short-term harms (2 weeks following vaccination) will be reported. The following outcomes are listed as primary and secondary under all the above categories:

\section{Primary outcomes}

1. Individuals with influenza-like illness symptoms, and a laboratory-confirmed diagnosis of influenza; that is, the incidence or prevalence of laboratory-confirmed influenza infection using any of the available diagnostic tests (RT-PCR or other laboratory tests) $;{ }^{9} 35$

2. Asthma exacerbations

\section{Secondary outcomes}

1. Hospitalisation (asthma-related and all-cause)

2. Death (all-cause and respiratory illness)
3. Primary care consultation (asthma-related and acute respiratory illness, including influenza-like illness)

4. Respiratory illness (clinically diagnosed or by self-report)

5. Adverse events (asthma exacerbation and other local or systematic reactions). ${ }^{9} 3637$

\section{Search methods}

We will search the published literature from January 1970 to January 2016 for work investigating the effectiveness of influenza vaccination in people with asthma. This start date has been chosen because the earliest study by Bell $e t a l^{8}$ in 1978, regarding the vaccination of children with asthma with a killed influenza virus, was included in two previous reviews that were conducted in 1995 and 2000, respectively. ${ }^{39}{ }^{40}$ There will be no language limitation.

We will search the following biomedical databases: MEDLINE (Ovid) (see online supplementary appendix 1), EMBASE (Ovid), Cumulative Index to Nursing and Allied Health Literature (CINAHL), Cochrane Central Register of Controlled Trials (CENTRAL), Scopus, Cochrane Database of Systematic Reviews (CDSR), Web of Science Core Collection, ScienceDirect, WHO Library Information System (WHOLIS), Global Health Library and Chinese databases (CNKI, Wanfang and ChongQing VIP). Electronic searches will be complemented by manually searching all reference lists of published and unpublished studies and reviews for additional studies. In addition, forward citation search will be performed on all identified studies using Web of Science. Furthermore, unpublished or ongoing clinical trials will be searched in clinical trials registry databases using http://www. controlled-trials.com/, http://www.clinicalTrials.gov/ and the WHO International Clinical Trials Registry Platform (ICTRP) using http://www.who.int/ictrp/en/.

We will contact pharmaceutical companies that manufacture influenza vaccines used in the trials to identify other published or unpublished studies. Authors of the studies included in the review will be contacted in case additional information is needed. Specific search strategies will be developed for each database. Two reviewers will independently undertake searches. Any disagreements that arise between the two reviewers will be resolved by discussions, or by the involvement of a third reviewer, if needed.

\section{Risk of bias assessment}

Two reviewers will independently assess the risk of bias of each study. Any disagreements arising between the two reviewers will be resolved through discussion, and if there is still disagreement or doubt, by the involvement of a third independent reviewer.

\section{Randomised controlled studies}

The risk of bias of experimental studies will be assessed using the criteria outlined in the Cochrane Collaboration's tool for assessing risk of bias. ${ }^{41}$ Studies 
will be evaluated according to the following six domains (1) sequence generation; (2) allocation concealment; (3) blinding of participants and personnel, and outcome assessors; (4) outcome ascertainment; (5) selective outcome reporting; and (6) other bias. Risk of bias in included studies will be divided into three categories: high, low, or unclear.

\section{Observational and non-randomised controlled studies}

The Quality Assessment Tool for Quantitative Studies Dictionary developed by the Effective Public Health Practice Project (EPHPP) will be used for the evaluation of observational studies and non-randomised controlled studies. ${ }^{42}$ The EPHPP tool contains the following eight components: selection bias (external validity), study design (allocation bias), confounders, blinding (detection bias), data collection methods, withdrawals and dropouts (attrition bias), intervention integrity and analyses. The first six components are rated as strong, moderate, or weak. There is no rating for the last two components. The overall study rating will be judged as strong, moderate, or weak, based on the component ratings.

\section{Data extraction and management}

The data from the observational and experimental studies that are eligible for inclusion after having the full text reviewed, will be extracted by two reviewers, independently, using a data abstraction form designed for this review. Any disagreement on the extraction of data of the included studies will be resolved through discussion or by the involvement of a third reviewer. The data extraction form will include general information for each study, population characteristics, intervention, outcomes, analysis and extra useful information.

\section{Data analysis}

Separate meta-analyses will be performed for clinically and methodologically comparable experimental and observational studies, respectively, to estimate the incidence or frequency of influenza infection and other influenza-related complications. Fixed-effects, or random-effects models, will be used to summarise the findings depending on the degree of clinical heterogeneity of the studies.

For dichotomous outcomes, the treatment effect will be estimated using risk ratio (RR) with $95 \%$ CIs or OR with $95 \%$ CIs. In addition, we will report absolute measures (eg, ARR, NNV, NNH) where they have been used.

Crossover and parallel trials will be included in this review. We will include crossover studies that assess shortterm safety in the relevant short-term safety meta-analysis. These will be analysed appropriately, taking the design into account.

Statistical heterogeneity will be assessed using the standard $\chi^{2}$ test and the $\mathrm{I}^{2}$ statistic, which describes that the proportion of dispersion across studies is due to true heterogeneity rather than to a sampling error (where no heterogeneity is represented by $0 \%$ and the highest level of heterogeneity is $100 \%)$. Significant $(50 \%)$ statistical heterogeneity will be investigated by conducting relevant subgroup and sensitivity analyses (see below) ${ }^{41}$

In the case where a study is eligible to be included in the review, but has missing data, authors will be contacted for provision of further data.

All statistical analyses will be undertaken using $\mathrm{R}$.

\section{Subgroup analyses}

Possible sources of heterogeneity in this review will be investigated with the following potential comparisons, if enough data are available from the included studies in this review:

1. Type of influenza vaccine: Trivalent Inactivated Vaccine (TIV) against Live Attenuated Influenza Vaccine (LAIV) $;^{43}$

2. Age of participants: People vaccinated for seasonal influenza aged 65 years or older against those less than 65 years old; ${ }^{44}$

3. Severity of asthma: people with mild to moderate asthma, compared with those with severe asthma; ${ }^{45} 46$

4. Immunity status: individuals with previous influenza vaccination against those with no previous influenza vaccination; ${ }^{12}$

5. Influenza-season mismatch: influenza seasons with antigenic match compared with seasons with mismatch; ${ }^{12}$

6. Influenza season-predominant strain: influenza season with dominant strains $\mathrm{A} / \mathrm{H} 1 \mathrm{~N} 1, \mathrm{~A} / \mathrm{H} 3 \mathrm{~N} 2$ and $\mathrm{B} ;{ }^{12}$

7. Time elapsed between vaccination and influenza infection: people developed influenza (diagnostically confirmed) in less than 14 days after being vaccinated against those with influenza after 14 days of their vaccination performed. ${ }^{12}$

\section{Sensitivity analyses}

If the number of studies and data available allow, sensitivity analysis will be undertaken with regards to study quality. The analysis will be restricted to low risk of bias trials, with adequate allocation generation, allocation concealment and blinding. For example, unblinded trials will not be included in the analysis. In addition, observational studies at low risk of bias according to the EPHPP quality assessment tool will be included in the analysis.

In addition, we will investigate the effect of comorbidities in people with asthma as a sensitivity analysis (where they have been reported).

\section{Publication bias}

Publication bias will be explored by performing funnel plots (where sufficient data are available).

\section{Grading the quality of evidence}

The quality of the body of evidence for each outcome will be assessed using the Grading of Recommendations 
Assessment, Development and Evaluation (GRADE) working group methodology. ${ }^{47}$ The evaluation is based on the following five domains: risk of bias, consistency, directness, precision, and publication bias. There are four levels of quality: high, moderate, low, and very low. The GRADE summary of findings tables will be developed, and we will present the quality of all primary and secondary outcomes.

\section{ETHICS AND DISSEMINATION}

Formal ethical approval is not required, as primary data will not be collected. The findings will be disseminated in peer-reviewed journals and conference presentations.

\section{CONCLUSION}

This systematic review will provide evidence regarding the effectiveness of influenza vaccination in people with asthma. The evidence will be obtained by searching, critically appraising, and synthesising the evidence from experimental and observational studies of published and unpublished literature.

\section{Registration and publishing statement}

The systematic review protocol will be registered with the PROSPERO International Prospective Register of Systematic Reviews (http://www.crd.york.ac.uk/prospero), and reported using Preferred Reporting Items for Systematic Reviews and Meta-analysis checklist to guide the reporting of the review. The systematic review will be shared with others. ${ }^{48}$

\section{Author affiliations}

${ }^{1}$ Asthma UK Centre for Applied Research, Usher Institute of Population Health Sciences and Informatics, The University of Edinburgh, Teviot, Medical School, Edinburgh, UK

${ }^{2}$ Nuffield Department of Primary Care Health Sciences, Oxford University, New Radcliffe House, Radcliffe Observatory Quarter, Oxford, UK

${ }^{3}$ Cardiff University, Institute of Primary Care and Public Health, Cardiff, UK ${ }^{4}$ Health Protection Scotland, NHS National Services Scotland, Meridian Court, Glasgow, UK

${ }^{5}$ Centre of Academic Primary Care, University of Aberdeen, Aberdeen, UK ${ }^{6}$ The University of Edinburgh, Teviot, Medical School, Edinburgh, UK

Contributors EV wrote this review. AS and CS contributed to conceiving this review, and commented critically on several drafts of the protocol. CB contributed to defining the study question and planning the review, and commented critically on several drafts of the protocol. BvW, JM and LR contributed on a draft of this review with critical comments. LT contributed in the creation of the early drafts of this review.

Funding The work was funded by the Chief Scientist Office of the Scottish Government under grant (AUKCAR/14/03). This work is carried out with the support of the Asthma UK Centre for Applied Research (AUK-AC-2012-01).

Competing interests None declared.

Provenance and peer review Not commissioned; externally peer-reviewed.

Open Access This is an Open Access article distributed in accordance with the Creative Commons Attribution Non Commercial (CC BY-NC 4.0) license, which permits others to distribute, remix, adapt, build upon this work noncommercially, and license their derivative works on different terms, provided the original work is properly cited and the use is non-commercial. See: http:// creativecommons.org/licenses/by-nc/4.0/

\section{REFERENCES}

1. Nair H, Brooks WA, Katz M, et al. Global burden of respiratory infections due to seasonal influenza in young children: a systematic review and meta-analysis. Lancet 2011;378:1917-30.

2. Centers for Disease Control and Prevention. Flu season. http://www. cdc.gov/flu/about/season/flu-season.htm (accessed 10 Mar 2015).

3. Thompson WW, Shay DK, Weintraub E, et al. Mortality associated with influenza and respiratory syncytial virus in the United States. JAMA 2003;289:179-86.

4. Molinari NA, Ortega-Sanchez IR, Messonnier ML, et al. The annual impact of seasonal influenza in the US: measuring disease burden and costs. Vaccine 2007;25:5086-96.

5. Eliakim-Raz N, Vinograd I, Zalmanovici Trestioreanu A, et al. Influenza vaccines in immunosuppressed adults with cancer. Cochrane Database Syst Rev 2013;10:CD008983.

6. Wong KK, Jain S, Blanton L, et al. Influenza-associated pediatric deaths in the United States, 2004-2012. Pediatrics 2013;132:796-804.

7. Khetsuriani N, Kazerouni NN, Erdman DD, et al. Prevalence of viral respiratory tract infections in children with asthma. J Allergy Clin Immunol 2007:119:314-21.

8. Glezen WP. Clinical practice. Prevention and treatment of seasonal influenza. N Engl J Med 2008;359:2579-85.

9. Public Health England. Influenza: the green book, chapter 19 https://www.gov.uk/government/uploads/system/uploads/attachment data/file/385226/Green_Book_Chapter_19_v8_2.pdf (accessed 10 Mar 2015)

10. Global Initiative for Asthma. Pocket Guide for Asthma Management and Prevention. http://www.ginasthma.org/documents/1/Pocket-Guidefor-Asthma-Management-and-Prevention (accessed 29 May 2015).

11. Centers for Disease Control and Prevention (CDC). Flu and People with Asthma. http://www.cdc.gov/flu/asthma/index.htm (accessed 23 Nov 2015).

12. Methods for measuring Influenza vaccine effectiveness during influenza seasons and pandemics in the European Union. https:// sites.google.com/site/epiflu/docu (accessed 17 Mar 2015).

13. Centers for Disease Control and Prevention (CDC). Prevention and control of seasonal influenza with vaccines. Recommendations of the Advisory Committee on Immunization Practices-United States, 2013-2014. MMWR Recomm Rep 2013;62(RR-07):1-43.

14. National Advisory Committee on Immunization (NACl). Statement on Seasonal Influenza Vaccine for 2013-2014. http://www.phac-aspc. gc.ca/publicat/ccdr-rmtc/13vol39/acs-dcc-4/index-eng.php (accessed 17 Mar 2015)

15. Background Paper on Influenza Vaccination and Immunization, SAGE Working Group. http://www.who.int/immunization/sage/ meetings/2012/april/1_Background_Paper_Mar26_v13_cleaned.pdf (accessed 14 Sep 2015)

16. Mereckiene J, Cotter S, Nicoll A, et al, The VENICE project gatekeepers group. Seasonal influenza immunisation in Europe. Overview of recommendations and vaccination coverage for three seasons: pre-pandemic (2008/09), pandemic (2009/10) and post-pandemic (2010/11). Euro Surveill 2014:19:pii $=20780$

17. British Thoracic Society. Scottish Intercollegiate Guidelines Network. British guideline on the management of asthma. A national clinical guideline. Revised 2014. https://www.brit-thoracic.org.uk/ document-library/clinical-information/asthma/ btssign-asthma-guideline-2014/ (accessed 23 Nov 2015).

18. Jefferson T, Rivetti A, Harnden A, et al. Vaccines for preventing influenza in healthy children. Cochrane Database Syst Rev 2008;(2): CD004879.

19. Beyer WE, de Bruijn IA, Palache AM, et al. Protection against influenza after annually repeated vaccination: a meta-analysis of serologic and field studies. Arch Intern Med 1999;159:182-8.

20. Dharmaraj $P$, Smyth RL. Vaccines for preventing influenza in people with cystic fibrosis. Cochrane Database Syst Rev 2014;3:CD001753.

21. Hicks KL, Chemaly RF, Kontoyiannis DP. Common community respiratory viruses in patients with cancer: more than just "common colds". Cancer 2003;97:2576-87.

22. Shovelton C. Research capability programme- background, vision, overview and glossery. NHS Connecting for Health, 2008.

23. Darvishian M, Bijlsma MJ, Hak E, et al. Effectiveness of seasonal influenza vaccine in community-dwelling elderly people: a meta-analysis of test-negative design case-control studies. Lancet Infect Dis 2014;14:1228-39.

24. Lone NI, Simpson C, Kavanagh K, et al. Seasonal Influenza Vaccine Effectiveness in the community (SIVE): protocol for a cohort study exploiting a unique national linked data set. BMJ Open 2012;2:e001019.

25. Simpson CR, Ritchie LD, Robertson C, et al. Effectiveness of H1N1 vaccine for the prevention of pandemic influenza in Scotland, UK: a retrospective observational cohort study. Lancet Infect Dis 2012;12:696-702. 
26. Hak E, Verheij TJM, Grobbee DE, et al. Confounding by indication in non-experimental evaluation of vaccine effectiveness: the example of prevention of influenza complications. J Epidemiol Community Health 2002;56:951-5.

27. Osterholm MT, Kelley NS, Sommer A, et al. Efficacy and effectiveness of influenza vaccines: a systematic review and meta-analysis. Lancet Infect Dis 2012;12:36-44.

28. Jefferson T, Rivetti A, Di Pietrantonj C, et al. Vaccines for preventing influenza in healthy children. Cochrane Database Sys Rev 2012;8 CD004879.

29. Jefferson T, Di Pietrantonj C, Rivetti A, et al. Vaccines for preventing influenza in healthy adults. Cochrane Database Sys Rev 2010;(7): CD001269.

30. Doshi P. Influenza: marketing vaccine by marketing disease. $B M J$ 2013;346:\{3037.

31. Jackson ML, Nelson JC. The test-negative design for estimating influenza vaccine effectiveness. Vaccine 2013;31:2165-8.

32. Foppa IM, Haber M, Ferdinands JM, et al. The case test-negative design for studies of the effectiveness of influenza vaccine. Vaccine 2013;31:3104-9.

33. De Serres G, Skowronski DM, Wu XW, et al. The test-negative design: validity, accuracy and precision of vaccine efficacy estimates compared to the gold standard of randomised placebo-controlled clinical trials. Euro Surveill 2013;18:20585.

34. Cates CJ, Rowe BH. Vaccines for preventing influenza in people with asthma. Cochrane Database Sys Rev 2013;2:CD000364.

35. Harper SA, Bradley JS, Englund JA, et al. Seasonal influenza in adults and children--diagnosis, treatment, chemoprophylaxis, and institutional outbreak management: clinical practice guidelines of the Infectious Diseases Society of America. Clin Infect Dis 2009;48:1003-32.

36. Vellozzi C, Burwen DR, Dobardzic A, et al. Safety of trivalent inactivated influenza vaccines in adults: background for pandemic influenza vaccine safety monitoring. Vaccine 2009;27:2114-20.

37. Hambidge SJ, Glanz JM, France EK, et al. Safety of trivalent inactivated influenza vaccine in children 6 to 23 months old. JAMA 2006;296:1990-7.
38. Bell TD, Chai H, Berlow B, et al. Immunization with killed influenza-virus in children with chronic asthma. Chest 1978;73:140-5.

39. Rothbarth PH, Kempen BM, Sprenger MJ. Sense and nonsense of influenza vaccination in asthma and chronic obstructive pulmonary disease. Am J Respir Crit Care Med 1995;151:1682-5; discussion 5-6.

40. Cates CJ, Jefferson TO, Bara Al, et al. Vaccines for preventing influenza in people with asthma (Cochrane Review). Cochrane Database Sys Rev 2000;(4):Cd000364.

41. Higgins JPT, Green S, eds. Cochrane handbook for Systematic Reviews of Interventions Version 5.1.0 [updated March 2011]. The Cochrane Collaboration. http://handbook.cochrane.org/ (accessed 8 May 2015).

42. Quality Assessment Tool for Quantitative Studies. Effective Public Health Practice Project. http://www.ephpp.ca/tools.html (accessed 8 May 2015).

43. Fleming DM, Crovari P, Wahn U, et al. Comparison of the efficacy and safety of live attenuated cold-adapted influenza vaccine, trivalent, with trivalent inactivated influenza virus vaccine in children and adolescents with asthma. Pediatr Infect Dis J 2006;25:860-9.

44. Jefferson T, Di Pietrantonj C, Al-Ansary LA, et al. Vaccines for preventing influenza in the elderly. Cochrane Database Sys Rev 2010;(2):CD004876.

45. Abadoğlu O, Mungan MDD, Paşaoglu GP, et al. Influenza vaccination in patients with asthma: Effect on the frequency of upper respiratory tract infections and exacerbations. $J$ Asthma 2004;41:279-83.

46. Global Strategy for Asthma Management and Prevention, Global Initiative for Asthma (GINA) 2015. http://www/ginasthma.org/ (accessed 29 May 2015).

47. Guyatt GH, Oxman AD, Vist GE, et al. GRADE: an emerging consensus on rating quality of evidence and strength of recommendations. BMJ 2008;336:924-6.

48. Moher $\mathrm{D}$, Liberati $\mathrm{A}$, Tetzlaff $\mathrm{J}$, et al. Preferred reporting items for systematic reviews and meta-analyses: the PRISMA statement. J Clin Epidemiol 2009;62:1006-12. 